
зрительных путей

\author{
Баев А.А. ${ }^{1}$ Погосбекян Э.Л. • Захарова Н.Е.' • Пицхелаури Д.И. • Баталов А.И. • Шкатова А.М. ${ }^{1}$ • \\ Шульц Е.И. • Быканов А.Е. • Маряшев С.А. ${ }^{1}$ ' Конакова Т.А. ${ }^{1}$ ' Пронин И.Н.'
}

Обоснование. Использование магнитно-резонансной (МР)-трактографии в нейрохирургии становится все более частой практикой благодаря возможности неинвазивно визуализировать проводящие пути белого вещества. Самый распространенный метод реконструкции трактов - детерминистический алгоритм диффузионно-тензорной МР-томографии. Однако этот метод построения проводящих путей имеет целый ряд существенных ограничений. К наиболее важным из них относятся отсутствие возможности визуализации пересекающихся между собой волокон, сложность построения трактов в области перифокального отека и в непосредственной близости к границам опухоли. Этих недостатков помогает избежать метод МР-трактографии, основанный на получении диффузионного изображения с высоким угловым разрешением (англ. high angulation reconstruction diffusion imaging, HARDI) с использованием алгоритма разложения по сферическим функциям (англ. constrained spherical deconvolution, CSD) для постобработки данных. Относительно недавно был предложен новый алгоритм для обработки данных HARDI: разложение MP-сигнала нескольких типов ткани мозга по сферическим функциям с использованием одного b-фактора - SS3T-CSD (single-shell 3-tissue CSD). Предположительно, он позволит улучшить построение проводящих путей в области перифокального отека или отека-инфильтрации. Цель - исследовать возможности алгоритма SS3T-CSD по сравнению с ST-CSD (single-tissue CSD - разложение MP-сигнала одного типа ткани мозга по сферическим функциям) при визуализации зрительной радиации и зрительных трактов у пациентов с глиомами.

Материал и методы. Десяти пациентам с впервые выявленными глиомами головного мозга выполняли диффузионную и рутинную МР-томографию головного мозга с последующей реконструкцией зрительной лучистости и зрительных трактов. Мы сравнили новые алгоритмы постобработки MP-трактографии STCSD и SS3T-CSD при визуализации зрительных трактов и зрительной лучистости у пациентов с глиомами головного мозга, поражающими различные отделы зрительного анализатора.

Результаты. Метод SS3T-CSD показал меньший средний процент ложноположительных трактов по сравнению с методом ST-CSD: 19,75 против 80,32\% в случаях близкого расположения опухоли к трактам и 5,27 против 25,27\% в случаях построения трактов в нормальном белом веществе.

Заключение. Метод SS3T-CSD имеет ряд преимуществ по сравнению с ST-CSD и позволяет успешно визуализировать зрительные проводящие пути, имеющие сложную структуру и неоднократно меняющие направление по своему ходу.

Ключевые слова: магнитно-резонансная трактография, зрительная лучистость, зрительные тракты, CSD-HARDI

Для цитирования: Баев АА, Погосбекян ЭЛ, Захарова НЕ, Пицхелаури ДИ, Баталов АИ, Шкатова АМ, Шульц ЕИ, Быканов АЕ, Маряшев СА, Конакова ТА, Пронин ИН. Магнитно-резонансная трактография на основе вероятностных алгоритмов разложения по сферическим функциям у пациентов с глиомами зрительных путей. Альманах клинической медицины. 2021;49(1):11-20. doi: 10.18786/2072-0505-2021-49-009.

Поступила 22.12.2020; доработана 17.02.2021; принята к публикации 19.02.2021; опубликована онлайн 12.03.2021 
$\mathrm{O}$ дна из важнейших задач нейрохирургии - радикальное удаление всего объема опухоли с максимальным сохранением функций соответствующего отдела мозга, избегая возникновения персистирующего неврологического дефицита, который может быть вызван повреждением здоровых тканей, как сохранных в пределах опухоли, так и ее окружающих. В этом плане большую сложность представляют опухоли глиального ряда, характеризующиеся инфильтративным ростом. При этом окружающие опухоль проводящие пути белого вещества головного мозга могут быть смещены за счет масс-эффекта, частично инфильтрированы или даже разрушены в случае полной инфильтрации [1]. Именно поэтому крайне важно определить в области самой опухоли, а также в непосредственной близости от нее расположение функционально значимых трактов белого вещества, которые должны быть сохранены во время операции. И этот вопрос должен решаться индивидуально для конкретного клинического случая.

Диффузионно-тензорная магнитнорезонансная томография (МРТ) - наиболее часто используемый метод нейрохирургического предоперационного планирования, позволяющий неинвазивно картировать проводящие пути белого вещества [2,3]. Этот метод уже давно интегрирован в нейрохирургическую клинику и довольно широко применяется в современных нейронавигационных системax [3]. При построении проводящих путей в большинстве клинических МРТ-приложений используется детерминистическая модель реконструкции трактов на основе диффузионно-тензорной МРТ. С помощью этой модели в каждом вокселе возможно оценить только одно направление волокна. Ранее было доказано, что до 90\% вокселей белого вещества в головном мозге содержат пересекающиеся волокна [4]. Единственная ориентация в таких вокселях белого вещества, полученная с помощью диффузионно-тензорной МРТ, может привести к визуализации анатомически неправдоподобных трактов и даже показать отсутствие трактов, то есть дает ложноотрицательные результаты $[5,6]$. Следствием картирования ложноположительных трактов белого вещества может стать частичная хирургическая резекция опухоли, что снизит выживаемость пациентов после нейрохирургических вмешательств. Ложноотрицательные результаты, наоборот, могут послужить причиной избыточного удаления опухоли с захватом здоровой ткани мозга, что ведет к последующим необратимым неврологическим нарушениям [7].

Для решения проблемы визуализации пересекающихся волокон было разработано несколько алгоритмов. Один из них - разложение по сферическим функциям (англ. constrained spherical deconvolution, CSD). CSD - вероятностный
Баев Александр Александрович - врач-рентгенолог отделения рентгеновских и радиоизотопных методов диагностики'; ORCID: https://orcid.org/0000-0003-49080534

$\triangle 125047$, г. Москва, ул. 4-я Тверская-Ямская, 16, Российская Федерация. E-mail: abaev@nsi.ru

Погосбекян Эдуард Леонидович - медицинский физик отделения рентгеновских и радиоизотопных методов диагностики'; ORCID: https://orcid.org/00000002-4803-6948. E-mail: epogosbekyan@nsi.ru

Захарова Наталья Евгеньевна - д-р мед. наук, профессор РАН, вед. науч. сотр. отделения рентгеновских и радиоизотопных методов диагностики'; ORCID: https://orcid.org/0000-0002-05163613. E-mail: nzakharova@nsi.ru

Пицхелаури Давид Ильич - д-р мед. наук, вед. науч. сотр., заведующий 7-м нейрохирургическим отделением (глиальные опухоли) с группой «Химиотерапевтическое лечение больных с опухолями центральной нервной системы»'; ORCID: https://orcid. org/0000-0003-0374-7970.E-mail:dav@nsi.ru
Баталов Артем Игоревич - канд. мед. наук, мл. науч. сотр. отделения рентгеновских и радиоизотопных методов диагностики'; ORCID: https://orcid.org/00000002-8924-7346. E-mail: abatalov@nsi.ru

Шкатова Анастасия Михайловна - врач-нейрохирург 7-го нейрохирургического отделения (глиальные опухоли) с группой «Химиотерапевтическое лечение больных с опухолями центральной нервной системы»'; ORCID: https://orcid.org/0000-0003-4957-4286.

E-mail: shkatova.a.m.v@gmail.com

Шульц Евгений Игоревич - канд. мед. наук, врач-рентгенолог отделения рентгеновских и радиоизотопных методов диагностики'; ORCID: https://orcid.org/0000-0001-5406-944X. E-mail: evgshults@gmail.com

Быканов Андрей Егорович - канд. мед. наук, врач-нейрохирург 7-го нейрохирургического отделения (глиальные опухоли) с группой

«Химиотерапевтическое лечение больных с опухолями центральной нервной системы»'; ORCID: https://orcid. org/0000-0002-0588-4779. E-mail: abykanov7@gmail.ru
Маряшев Сергей Алексеевич - д-р мед. наук, врач-нейрохирург 7-го нейрохирургического отделения (глиальные опухоли) с группой «Химиотерапевтическое лечение больных с опухолями центральной нервной системы»'; ORCID: https://orcid. org/0000-0002-0108-0677.

E-mail: smaryashev@gmail.com

Конакова Татьяна Александровна - аспирант отделения рентгеновских и радиоизотопных методов диагностики'; ORCID: https://orcid.org/0000-0002-25057981.E-mail: tknsipet@gmail.com

Пронин Игорь Николаевич - д-р мед. наук, профессор, академик РАН, заведующий отделением рентгеновских и радиоизотопных методов диагностики'; ORCID: https://orcid.org/0000-0002-44800275. E-mail: pronin@nsi.ru 
алгоритм реконструкции трактов на основе диффузионных изображений с высоким угловым разрешением (англ. high angulation reconstruction diffusion imaging, HARDI) [8]. Было показано, что такой подход дает лучшие результаты по сравнению со стандартной диффузионно-тензорной моделью [8].

Существует несколько вариантов вероятностных алгоритмов обработки HARDI: разложение МР-сигнала одного типа ткани мозга по сферическим функциям (англ. single-tissue CSD, ST-CSD) [9], разложение MP-сигнала нескольких типов ткани мозга по сферическим функциям с использованием нескольких b-факторов (англ. multi-shell multi-tissue CSD, MSMT-CSD) [10] и разложение MP-сигнала нескольких типов ткани мозга по сферическим функциям с использованием одного b-фактора (англ. single-shell 3-tissue CSD, SS3T-CSD) [2]. Алгоритм MSMT-CSD по сравнению с другими двумя методами имеет недостаток - существенно большее время сканирования, поскольку для него нужны диффузионные МР-данные с несколькими b-факторами, а не с одним. В связи с этим мы не стали изучать алгоритм MSMTCSD в нашей работе, но исследовали возможности нового алгоритма SS3T-CSD по сравнению c ST-CSD при визуализации зрительной радиации и зрительных трактов у пациентов с глиомами.

\section{Материал и методы}

В пилотное исследование были включены 10 пациентов с впервые выявленными глиомами головного мозга: 6 пациентов с глиомами низкой степени злокачественности, 2 - с анапластическими астроцитомами, 2 - с глиобластомами.

Всем пациентам перед удалением опухоли была выполнена МРТ головного мозга при помощи МР-томографа с магнитной индукцией 3,0 T (Signa HDxt, General Electric, США) и 8-канальной головной катушкой. Помимо рутинных последовательностей МРТ проводилось сканирование в режимах HARDI, T2 CUBE и T1 3D SPGR.

Были получены Т1-взвешенные анатомические изображения с изотропным вокселем $1 \times 1 \times 1$ мм. Режим HARDI имел следующие параметры: воксель $3 \times 3 \times 3$ мм, 54 среза, время повторения (англ. repetition time, TR) $=15000$ мс, время эхо (англ. echo time, ТЕ) = 110 мс, поле обзора (англ. field of view, FOV) $=240$ мм, 55 градиентных направлений для $\mathrm{b}=2000 \mathrm{c} / \mathrm{Mm}^{2}$.
Следует отметить, что все алгоритмы CSD чувствительны к шуму МР-сигнала. Эта проблема решается путем увеличения количества направлений диффузионного градиента при сканировании.

Выполняли следующие этапы постобработки диффузионных изображений:

1) конвертирование изображений из формата DICOM в Nifti;

2) удаление теплового шума и артефактов Гиббса с помощью утилит dwidenoise и mrdegibbs из программного пакета MRtrix3 (https://www. mrtrix.org/) [11];

3) коррекция движения и артефактов вихревых токов с помощью утилиты eddy из программного обеспечения FMRIB Software Library (FSL);

4) создание маски, исключающей внемозговые структуры, с помощью утилиты dwi2mask из программного пакета MRtrix3;

5) интерполяция диффузионного изображения до размера вокселя $1 \times 1 \times 1 \mathrm{Mm}^{3}$.

После этого Т1-взвешенное изображение было корегистрировано на интерполированное диффузионное изображение путем аффинных преобразований с помощью программы ANTs (http:// stnava.github.io/ANTs/).

\section{Алгоритм ST-CSD}

Согласно рекомендациям [12] для обработки ST-CSD использовались данные со значением $\mathrm{b}=2000$ с $/ \mathrm{мm}^{2}$. По диффузионным изображениям вычислялись все тракты, находящиеся внутри маски мозга. Для этого применяли программное обеспечение MRtrix3. Все параметры трактографии были оставлены по умолчанию, кроме следующих:

1) алгоритм вычисления трактов, предложенный в статье [9];

2) длина искомых трактов не должна превышать 200 мм;

3) количество искомых трактов не должно превышать 5 млн.

Как правило, количество найденных этим методом волокон составляло от 1 до 1,5 млн.

\section{Алгоритм SS3T-CSD}

Для обработки SS3T-CSD были использованы те же данные, что и для ST-CSD. Применяли программное обеспечение MRtrix3 с аналогичными параметрами, кроме алгоритма вычисления трактов (в этом случае использовали алгоритм, предложенный в статье [13]). Как правило, этим методом обнаруживалось от 800 тыс. до 1 млн волокон. 
Сравнение алгоритмов реконструкции проводящих путей зрительной радиации у 10 пациентов с глиомами головного мозга

\begin{tabular}{|c|c|c|c|c|c|c|}
\hline \multirow{2}{*}{$\begin{array}{c}\text { Пациент, } \\
\text { № } п / п\end{array}$} & \multirow[t]{2}{*}{ Диагноз } & \multirow{2}{*}{$\begin{array}{c}\text { Локализация } \\
\text { опухоли }\end{array}$} & \multicolumn{2}{|c|}{ Метод ST-CSD } & \multicolumn{2}{|c|}{ Метод SS3T-CSD } \\
\hline & & & $\begin{array}{l}\text { NOT_ROIs, } \\
\text { абc. }\end{array}$ & $\mathrm{FP} \%$ & $\begin{array}{l}\text { NOT_ROls, } \\
\text { aбc. }\end{array}$ & $\mathrm{FP} \%$ \\
\hline $1^{*}$ & Глиобластома & $\begin{array}{l}\text { Левая } \\
\text { височная } \\
\text { доля }\end{array}$ & 13 & 75,65 & 6 & 18,13 \\
\hline $2^{*}$ & Глиобластома & $\begin{array}{l}\text { Левая } \\
\text { затылочная } \\
\text { доля }\end{array}$ & 14 & 110,21 & 9 & 23,54 \\
\hline 3 & $\begin{array}{l}\text { Пилоидная } \\
\text { астроцитома }\end{array}$ & $\begin{array}{l}\text { Правый } \\
\text { зрительный } \\
\text { тракт }\end{array}$ & 10 & 17,23 & 5 & 2,57 \\
\hline 4 & $\begin{array}{c}\text { Анапластическая } \\
\text { астроцитома }\end{array}$ & $\begin{array}{l}\text { Правая } \\
\text { височная } \\
\text { доля }\end{array}$ & 11 & 36,23 & 6 & 7,43 \\
\hline $5^{*}$ & $\begin{array}{l}\text { Диффузная } \\
\text { астроцитома }\end{array}$ & $\begin{array}{l}\text { Правая } \\
\text { височная } \\
\text { доля }\end{array}$ & 15 & 65,32 & 8 & 19,32 \\
\hline 6 & Ганглиоглиома & $\begin{array}{l}\text { Правый } \\
\text { зрительный } \\
\text { тракт }\end{array}$ & 12 & 14,34 & 5 & 3,76 \\
\hline $7^{*}$ & $\begin{array}{l}\text { Пилоидная } \\
\text { астроцитома }\end{array}$ & $\begin{array}{l}\text { Левая } \\
\text { затылочная } \\
\text { доля }\end{array}$ & 7 & 60,21 & 5 & 17,43 \\
\hline 8 & $\begin{array}{l}\text { Пилоидная } \\
\text { астроцитома }\end{array}$ & $\begin{array}{l}\text { Правая } \\
\text { затылочная } \\
\text { доля }\end{array}$ & 8 & 43,32 & 4 & 8,34 \\
\hline 9 & Ганглиоглиома & $\begin{array}{l}\text { Правая } \\
\text { височная } \\
\text { доля }\end{array}$ & 7 & 15,23 & 4 & 4,23 \\
\hline $10^{*}$ & $\begin{array}{c}\text { Анапластическая } \\
\text { астроцитома }\end{array}$ & $\begin{array}{l}\text { Правая } \\
\text { височная } \\
\text { доля }\end{array}$ & 13 & 90,21 & 6 & 20,32 \\
\hline
\end{tabular}

FP\% - отношение числа ложноположительных трактов к числу построенных трактов, NOT_ROIs - количество использованных исключающих областей интереса

*Пациенты с опухолью, расположенной в непосредственной близости к зрительной лучистости или зрительному тракту

\section{Магнитно-резонансная трактография}

Во всех случаях выбор начальной области интереca (англ. region of interest, ROI) для реконструкции тракта основывался на анатомических данных MPT, которые были корегистрированы с изображениями HARDI. Такой подход значительно облегчает процесс отслеживания волокон и повышает общую надежность методики, поскольку позволяет осуществлять точный анатомический контроль реконструированных трактов.
Как показано на рис. 1, при МР-трактографии зрительной лучистости и зрительных трактов использовалась начальная ROI, покрывающая перекрест зрительных нервов, вторая ROI помещалась в область латерального коленчатого тела и третья - в медиальные отделы затылочной доли. Затем ставили дополнительные ROI для выполнения сегментации зрительной лучистости. На рис. 2 отображены компоненты зрительной лучистости для одного пациента. Анатомический ход петли Мейера, центрального и дорсального пучков, которые все вместе соединяют латеральное коленчатое тело с первичной зрительной корой, следует ожидаемым контурам, ход которых описан далее в разделе «Обсуждение». Полученные изображения трактов, реконструированные при помощи алгоритма SS3T-CSD, были совмещены c Т1-взвешенными МР-изображениями и использованы для интраоперационной навигационной системы Fiagon (Fiagon AG Medical Technologies). Для этого с помощью скрипта на MATLAB (MathWorks, США), написанного нашей группой, на Т1-взвешенном МР-изображении воксели, содержащие тракты, были затемнены. После этого модифицированное изображение Т1 было сохранено в формате DICOM.

Статистическая обработка результатов проводилась в программе Statistica 12 (StatSoft, США). С помощью статистического анализа для каждого пациента вычисляли отношение числа ложноположительных трактов (англ. false positive, FP) к числу «истинных» трактов, выраженное в процентах. Пациентов поделили на две группы: в первую группу вошли пациенты с опухолью, расположенной в непосредственной близости к зрительной лучистости или зрительному тракту, во вторую - остальные пациенты с опухолями. Затем для двух групп пациентов и для методов ST-CSD и SS3T-CSD посчитали средние значения FP. Методы сопоставили между собой, сравнив средние по критерию Стьюдента. В качестве критического уровня значимости выбрали $\mathrm{p}=0,05$.

\section{Результаты}

Результаты CSD-HARDI двух пациентов представлены на рис. 3 и 4. При сравнении двух алгоритмов расчета HARDI большее количество ложноположительных трактов отмечалось на трактограммах, полученных при помощи ST-CSD, в то время как на 2D-структурных картах, полученных методом SS3T-CSD, видно: тракты в сером веществе и ликворе практически отсутствуют, что отражает более корректное моделирование 

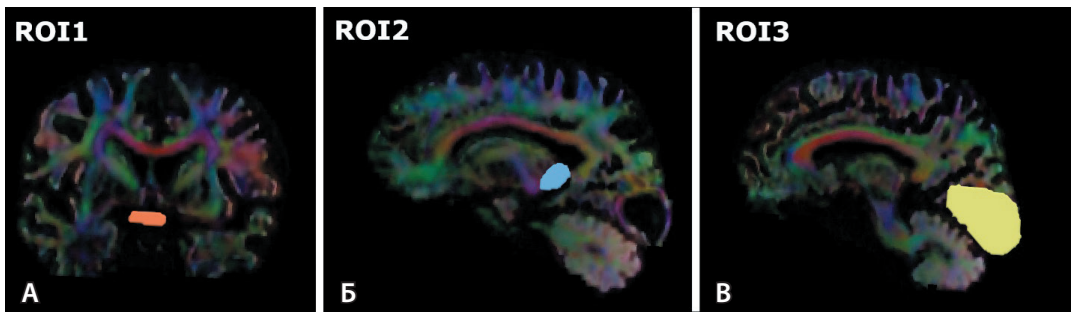

Рис. 1. Области интереса (ROI), выбранные на структурных картах с цветовым кодированием, где цвет указывает направление диффузии: A - ROl1, зона перекреста зрительных нервов (оранжевый цвет); $\mathbf{5}$ - ROI2, область латерального коленчатого тела (голубой цвет); В - ROI3, медиальные отделы затылочной доли (желтый цвет)
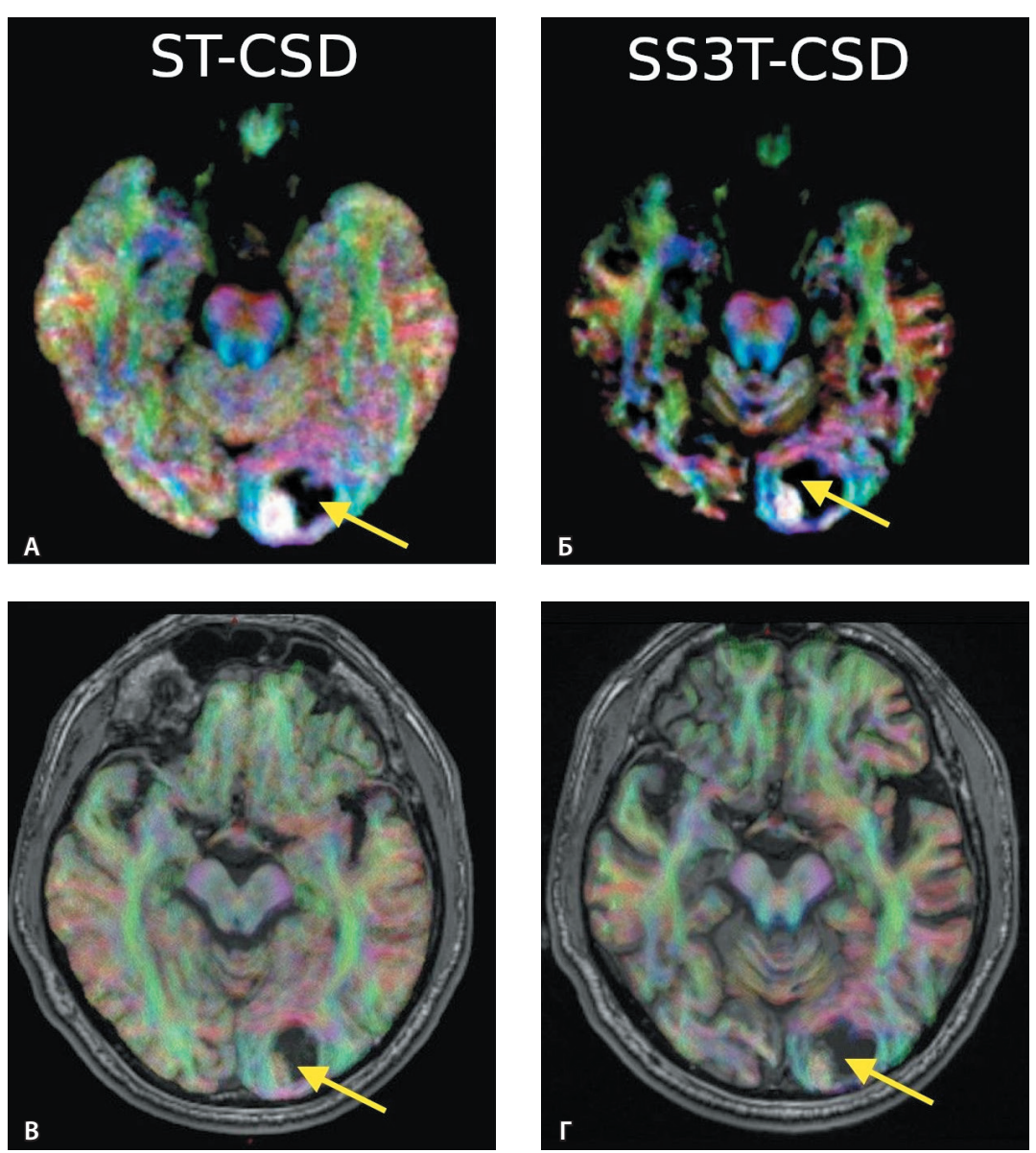

Рис. 3. Сравнение результатов ST-CSD (A, B) и SS3T-CSD (Б, Г) у пациента с глиобластомой затылочной доли: А, Б - 2D-структурные карты с цветовым кодированием, отражающие направление диффузии (красный цвет - комиссуральные волокна (слева направо), зеленый цвет - ассоциативные волокна (передне-заднее направление), синий цвет - проекционные волокна (верхне-нижнее направление)); В, Г - 2D-трактограммы, совмещенные с Т1-взвешенным изображением. Стрелка указывает на внутримозговую опухоль (глиобластому) в левой затылочной доле

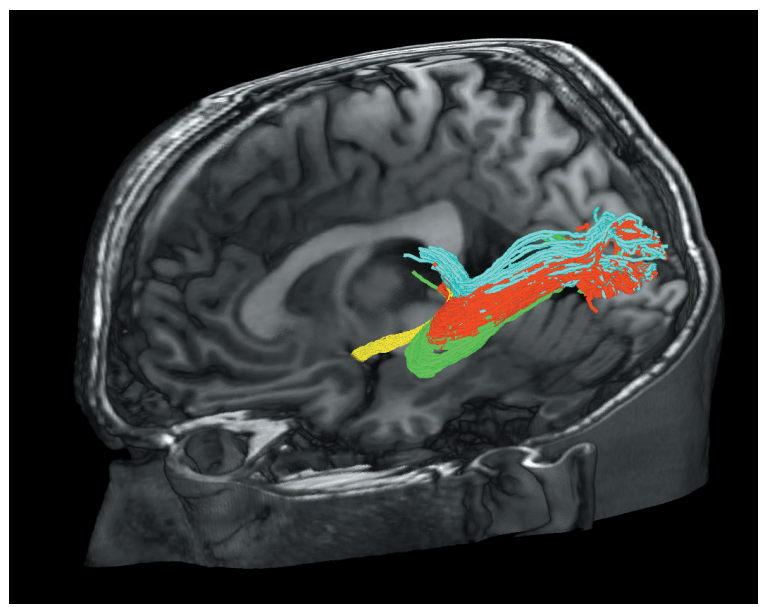

Рис. 2. Тракты зрительной лучистости: голубым цветом показан дорсальный сегмент, красным - центральный, зеленым - петля Мейера, желтым - зрительные тракты

сигнала методом SS3T-CSD в указанных структуpax.

C применением алгоритма SS3T-CSD нами была успешно реконструирована зрительная лучистость и зрительные тракты в полушариях головного мозга с двух сторон у всех 10 исследуемых пациентов. На рис. 5 и 6 показаны результаты построения зрительных трактов и зрительной лучистости на основании данных, полученных методами SS3T-CSD и ST-CSD.

В результате использования алгоритма SS3TCSD мы получили меньшее количество ложноположительных трактов, при этом во всех случаях данная методика позволила более надежно выделять разные компоненты зрительной лучистости. Для реконструкции зрительных трактов и зрительной лучистости требовалось применять не только ROI, через которые проходили искомые тракты (включающие), но и ROI, через которые проходили ложноположительные тракты (исключающие). В таблице для каждого пациента приведено количество использованных исключающих ROI (NOT_ROIs), а также отношение количества ложноположительных трактов к числу построенных, выраженное в процентах (FР\%). Отметим: оба метода дают большой процент ложноположительных трактов в случаях, когда опухоль расположена в непосредственной близости к зрительной лучистости или зрительному тракту, - среднее значение для метода STCSD составило 80,32\%, для SS3T-CSD - 19,75\% $(\mathrm{p}=0,0002)$.

В случаях, когда тракты строились в нормально выглядящем белом веществе, метод SS3T-CSD 

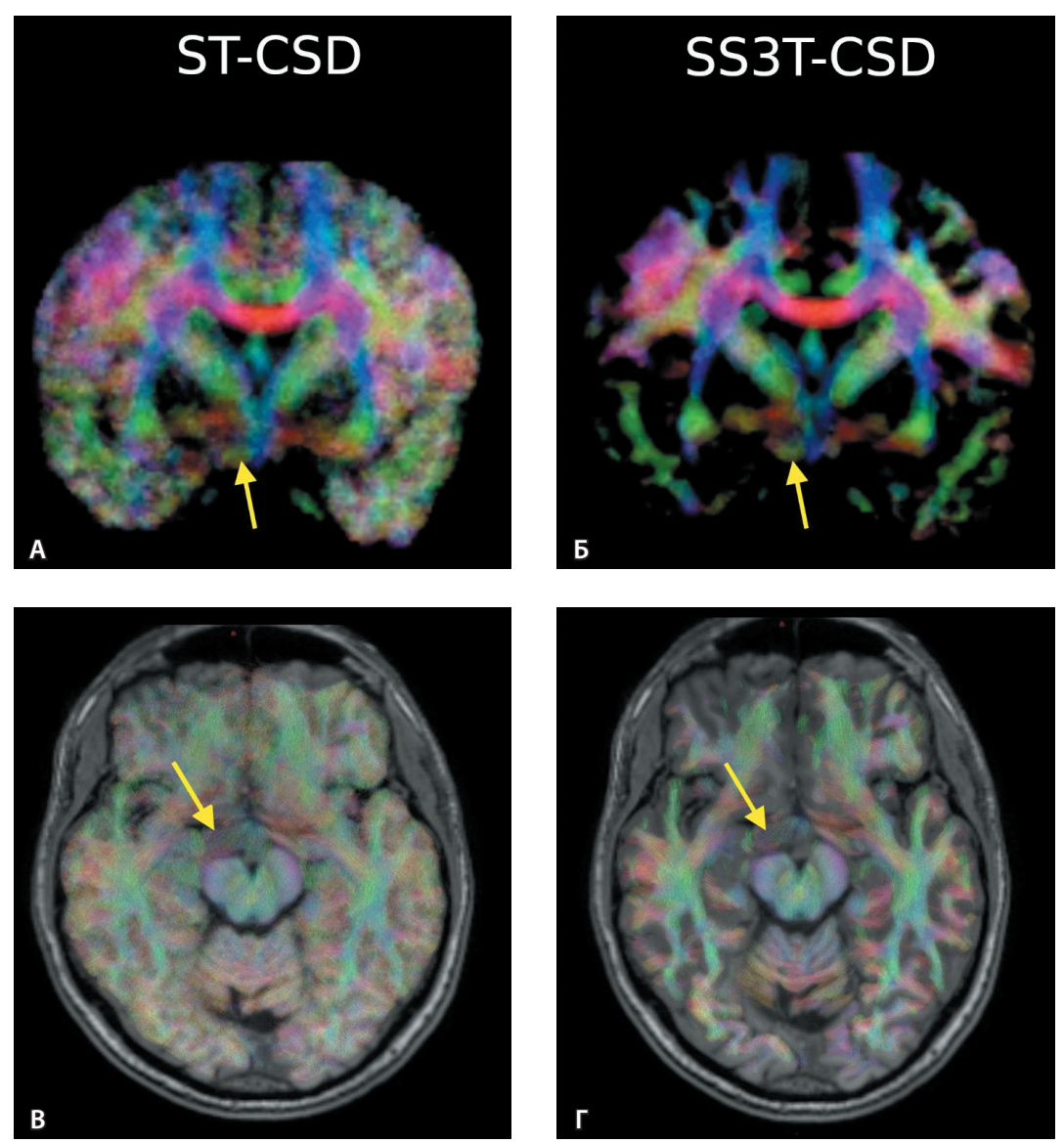

Рис. 4. Сравнение результатов ST-CSD (A, B) и SS3T-CSD (Б, Г) у пациента с пилоидной астроцитомой в области зрительного тракта справа: А, Б - 2D-структурные карты с цветовым кодированием, отражающие направление диффузии (красный цвет комиссуральные волокна (слева направо), зеленый цвет - ассоциативные волокна (передне-заднее направление), синий цвет - проекционные волокна (верхне-нижнее направление)); В, Г - 2D-трактограммы, совмещенные с Т1-взвешенным изображением. Стрелка указывает на опухоль в области правого зрительного тракта

демонстрировал существенно меньшее количество ложноположительных трактов: среднее значение для метода ST-CSD равнялось 25,27\% и для метода SS3T-CSD - 5,27\% (p=0,0116). Мы считаем, что такой процент ложноположительных трактов, полученных методом SS3T-CSD в здоровом белом веществе, не высокий и не критический для последующих интерпретаций и клинических решений. Таким образом, метод SS3T-CSD показал ряд преимуществ перед методом ST-CSD.

\section{Обсуждение}

В представленной работе мы исследовали возможности вероятностных алгоритмов реконструкции трактов ST-CSD и SS3T-CSD при визуализации зрительной радиации и зрительных путей у пациентов с глиомами.
Анатомически зрительные проводящие пути состоят из нескольких отделов, включающих в себя зрительные нервы, перекрест зрительных нервов, зрительные тракты, латеральные коленчатые тела и зрительную лучистость. Для нашей работы интерес представлял центральный отрезок зрительного анализатора - от перекреста зрительных нервов до коры шпорной борозды. Пациентов с поражением зрительных нервов не включали в протокол исследования в связи со сложностью реконструкции проводящих путей на фоне выраженных артефактов от костных структур орбитальной области.

Зрительный тракт представляет собой пучок нервных волокон, который соединяет зрительные нервы и хиазму с латеральными коленчатыми телами. Последние расположены в нижнелатеральных отделах таламусов и содержат третичные нейроны, образующие зрительную лучистость, волокна которой проецируются в серое вещество шпорной борозды затылочных долей - в область первичной зрительной коры, центральную часть зрительного анализатора [14].

Зрительная лучистость имеет три сегмента: тело (центральный сегмент), вентральный и дорсальный сегменты (рис. 7). Вентральный сегмент состоит из волокон, располагающихся в глубине средней височной извилины и образующих петлю над крышей височного рога, называемую петлей Мейера, которая, проходя над треугольником и затылочным рогом бокового желудочка, оканчивается в нижней шпорной коре. Петля Мейера расположена кзади от передней комиссуры и крючкообразного пучка, на 5 мм кпереди от переднего края височного рога и в среднем на 31 мм кзади от переднего края полюса височной доли [15]. Центральный сегмент зрительной лучистости (тело) проходит книзу от нижнего продольного пучка и продолжается в передне-заднем направлении до пересечения со слуховой лучистостью, выходящей из медиального коленчатого тела. Затем вместе они проходят медиальнее тапетума (части волокон мозолистого тела), где делают поворот на 90. Дорсальный сегмент зрительной лучистости направляется к коре затылочной доли выше или ниже шпорной борозды (первичная зрительная кора).

За долгий период, прошедший с момента первой работы, опубликованной А. Meyer в 1907 г. [16], было написано множество статей об анатомии зрительной лучистости, ее переднего или вентрального сегмента (петли Мейера), а также о зрительных нарушениях после операций на височных долях. 

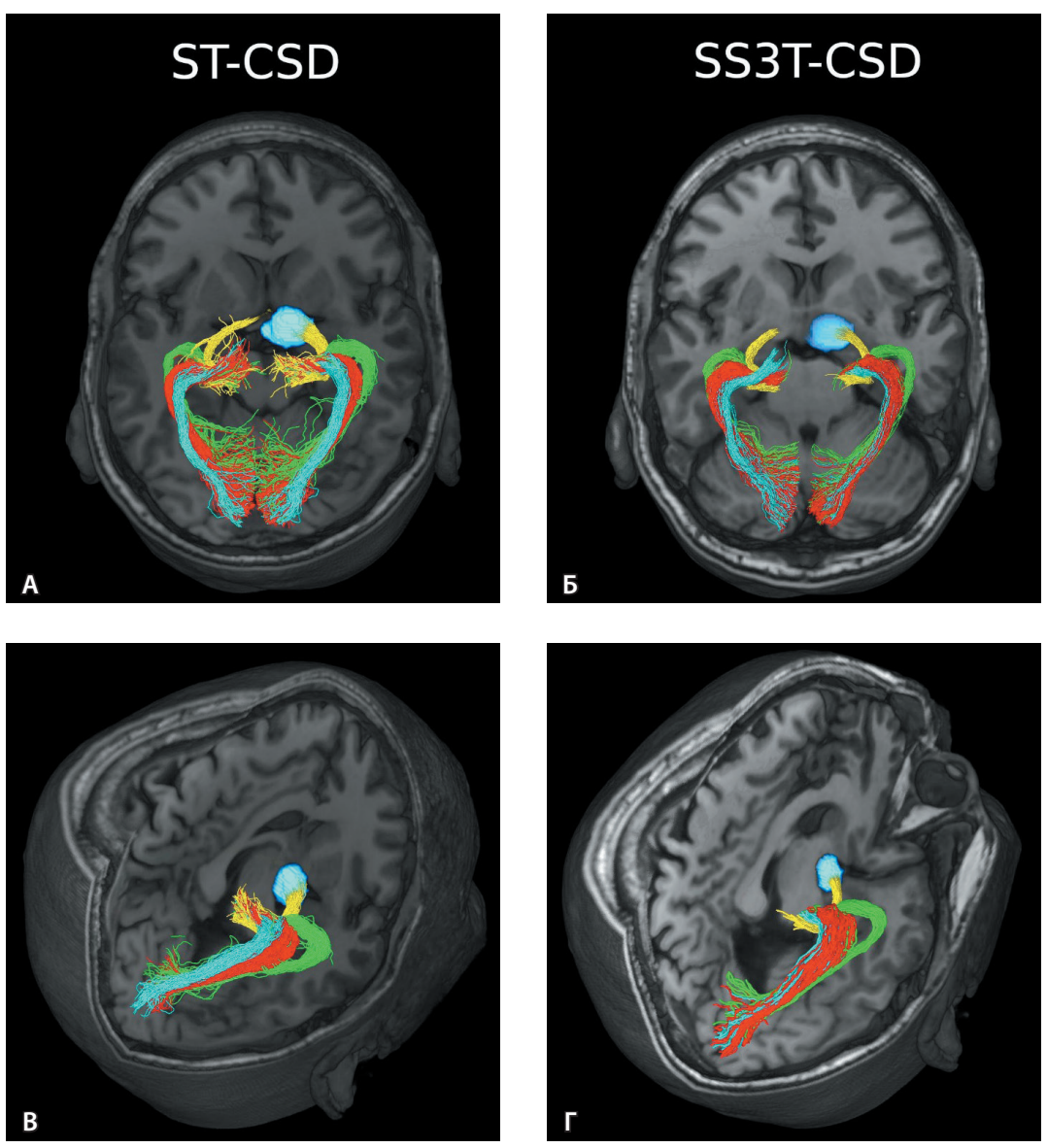

Рис. 5. Проводящие пути пациента с пилоидной астроцитомой зрительного тракта слева (A, B - ST-CSD; Б, Г - SS3T-CSD): петля Мейера выделена зеленым цветом, центральный сегмент зрительной лучистости - красным, дорсальный сегмент зрительной лучистости голубым, зрительные тракты - желтым. Магнитно-резонансная трактография позволяет определить полное включение зрительного тракта в структуру опухоли, что делает оперативное вмешательство с целью удаления новообразования невозможным

Из трех трактов - зрительная лучистость, крючкообразный тракт, нижний продольный пучок - образуется структура, называемая сагиттальным пластом (stratum). Сагиттальный пласт - конгломерат переплетенных между собой волокон белого вещества, ориентированных в сагиттальной плоскости, где волокна идут от височной доли к затылочной. E.H. Sincoff и соавт. [17], U. Türe и соавт. [18] установили, что волокна задней таламической ножки и нижнего лобно-затылочного тракта смешиваются между собой в сагиттальном пласте, что приводит к сложности их разделения и идентификации, в том числе определения точного хода волокон зрительной лучистости в их составе.

B анатомических работах U. Ebeling и Н.J. Reulen показано, что петля Мейера в большинстве случаев достигала переднего края височного рога, но в нескольких из 50 препаратов она не доходила до переднего края височного рога 5 мм [19]. P.A. Rubino и соавт., используя метод Клинглера и применяя операционный микроскоп и 3D-моделирование фотографий 20 замороженных срезов мозгового вещества, установили, что волокна вентральной порции зрительной лучистости, то есть волокна петли Мейера, достигали края височного рога во всех 20 препаратах нормального мозга человека. В каждом из них с помощью операционного микроскопа был выявлен очень тонкий слой передней части петли Мейера, которая во всех наблюдениях распространялась до края височного рога [15]. Во всех исследованных образцах зрительная лучистость полностью покрывала крышу височного рога и была отделена от нее только тонким слоем волокон тапетума. Описанные выше анатомические особенности расположения проводящих путей в стволе височной доли имеют решающее значение для двух нейрохирургических доступов: трансильвиевого доступа к медиальным отделам височной доли и височного трансоперкулярного доступа к темпоро-инсулярным глиомам. При обоих доступах происходит пересечение ствола височной доли, что особенно важно учитывать в доминантном полушарии [20].

Таким образом, зрительные пути представляют собой сложную и комплексную нейроанатомическую структуру, состоящую как из идущих параллельно, так и пересекающихся пучков нервных волокон, являющуюся критической при операциях на височной и затылочной долях мозга. Ее предоперационная визуализация на основе данных МР-трактографии - крайне сложная диагностическая задача и своеобразный вызов для нейрорадиологов.

Алгоритм ST-CSD [9] не предполагает информации о количестве волокон, представленных в вокселе, или об их пересечении, при этом используется модель со следующими упрощенными представлениями о белом веществе:

1) обмен внеаксональной и внутриаксональной воды в одном аксоне отсутствует;

2) радиус кривизны любых нервных пучков составляет больше нескольких десятков мкм;

3) диффузионные характеристики всех нервных пучков в мозге идентичны;

4) измеренный диффузионно-взвешенный сигнал в вокселе равен сумме сигналов от областей вокселя с разными направлениями диффузии. 

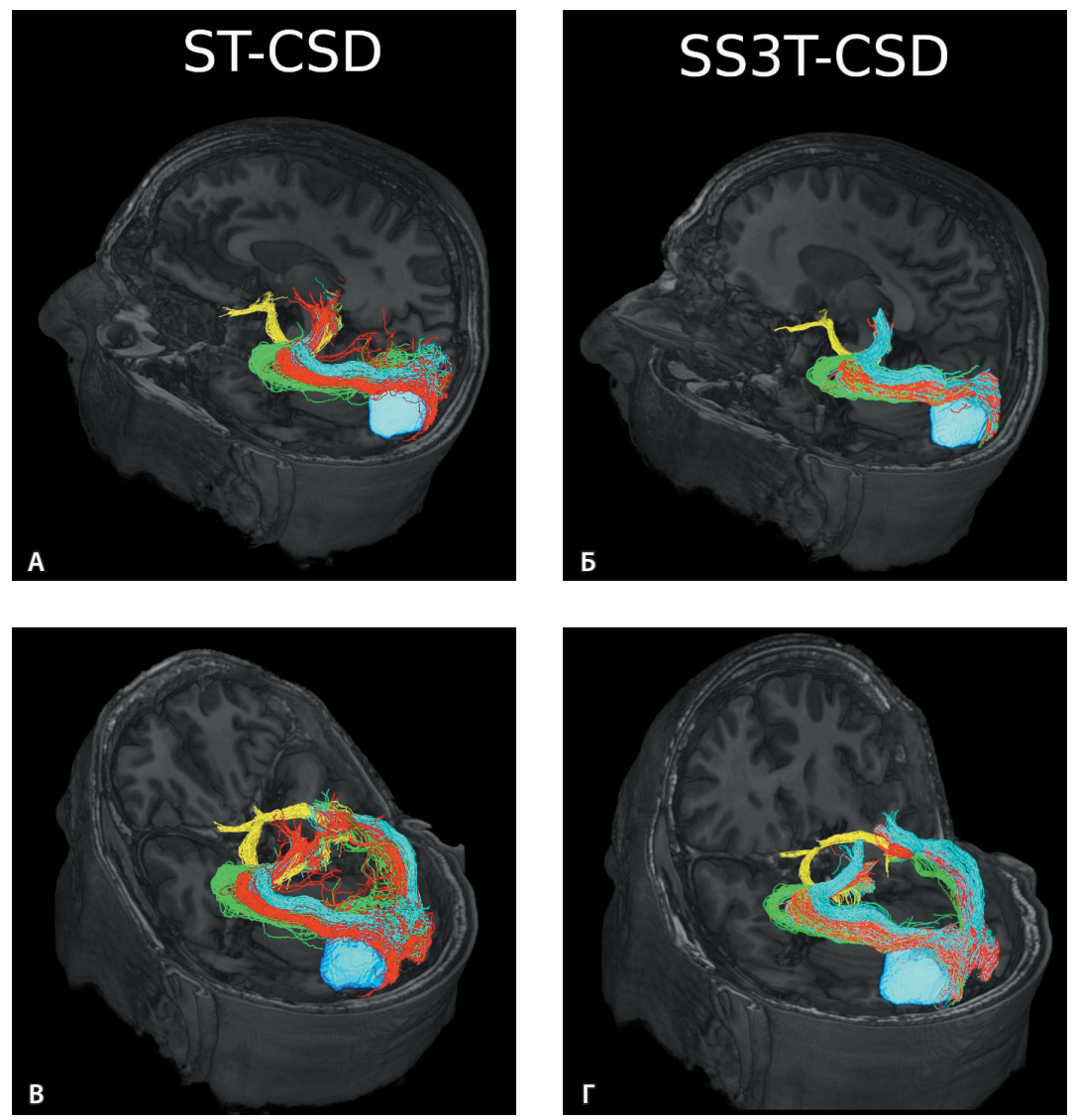

Рис. 6. Проводящие пути зрительной лучистости (А, В - ST-CSD; Б, Г - SS3T-CSD) по сегментам (выделены зеленым, красным и голубым цветом) и зрительного тракта (выделено желтым цветом) у пациента с глиобластомой в левой затылочной доле. Магнитнорезонансная трактография визуализирует дислокацию конечных отделов зрительных путей в проекции затылочной доли, что позволяет рассматривать показания для хирургического удаления новообразования

Новый алгоритм MSMT-CSD позволяет моделировать не только воксели белого вещества, но и воксели, содержащие серое вещество, а также спинномозговую жидкость [10]. По мнению авторов, это позволяет более точно реконструировать проводящие пути мозга. К главным недостаткам MSMT-CSD по сравнению с STCSD относится необходимость использования нескольких объемов с разными значениями b-фактора, обычно называемыми "multi-shell" данными, что приводит к значительному увеличению времени сканирования. Для того чтобы избежать использования нескольких объемов и увеличения времени исследования, был предложен метод SS3T-CSD [13, 21]. Он направлен на получение аналогичных MSMT-CSD результатов, но при этом используются только один объем и значение $\mathrm{b}=0$ для моделирования тканей мозга. В настоящее время метод SS3T-CSD

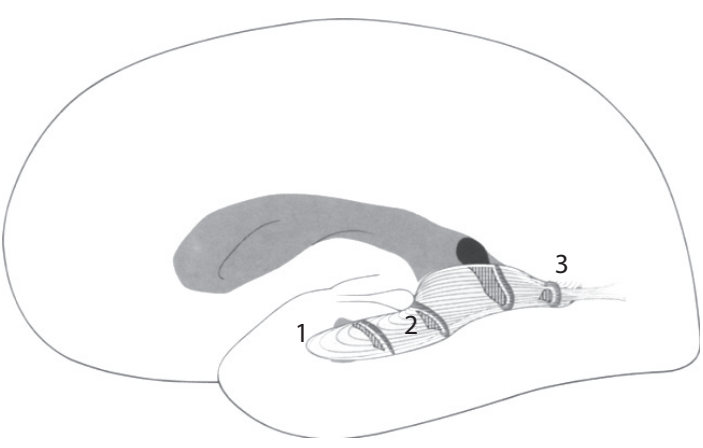

Рис. 7. Схема зрительной лучистости: 1 - вентральный сегмент (петля Мейера), 2 - центральный сегмент (тело), 3 - дорсальный сегмент

еще тестируется разработчиками (https://3tissue. github.io) и не входит в стабильную версию программы MRTrix3 (www.mrtrix.org).

Тем не менее, по нашему мнению, алгоритм реконструкции трактов SS3T-CSD имеет целый ряд преимуществ по сравнению с методом STCSD. Важным моментом следует признать отсутствие необходимости проведения отдельных измерений для каждого алгоритма: для обоих алгоритмов подходят одни и те же диффузионные данные без увеличения времени сканирования. Кроме того, метод SS3T-CSD показал более корректное моделирование сигнала в сером веществе и отсутствие МР-сигнала в ликворе, что позволяет получить более точную реконструкцию трактов белого вещества мозга.

Самыми сложными для интерпретации данных трактографии остаются области, где проводящие пути инфильтрированы опухолевой тканью. Для оценки корректности построения проводящих путей в таких условиях необходимо продолжить исследование с участием большего числа пациентов при сопоставлении с интраоперационными данными прямой электростимуляции проводящих путей, с выполнением контрольных исследований в послеоперационном периоде и клинической оценкой состояния пациента до и после операции.

\section{Заключение}

Метод SS3T-CSD имеет ряд преимуществ по сравнению с алгоритмом ST-CSD в построении проводящих путей белого вещества головного мозга и позволяет успешно визуализировать зрительные проводящие пути, имеющие сложную структуру и неоднократно меняющие направление по своему ходу. (ङ) 


\section{Дополнительная информация}

\section{Финансирование}

Работа проведена без привлечения дополнительного финансирования со стороны третьих лиц.

\section{Конфликт интересов}

Авторы декларируют отсутствие явных и потенциальных конфликтов интересов, связанных с публикацией настоящей статьи.

\section{Участие авторов}

А.А. Баев - концепция и дизайн исследования, обработка материала, анализ полученных данных, поиск и анализ литературы, написание текста; Э.Л. Погосбекян - анализ результатов, статистическая обработка данных, поиск и анализ литературы, написание текста; Н.Е. Захарова - концепция и дизайн статьи, анализ и интерпретация результатов исследования, написание и редактирование текста, утверждение итогового варианта текста рукописи; И.Н. Пронин - концепция и дизайн исследования, редактирование текста, утверждение итогового варианта текста рукописи; Д.И. Пицхелаури - концепция и дизайн статьи, сбор и обработка материала, анализ результатов; А.И. Баталов - сбор и обработка материала, редактирование текста; А.М. Шкатова - сбор и обработка материала, поиск и анализ литературы, написание текста; Е.И. Щульц, А.Е. Быканов, С.А. Маряшев и Т.А. Конакова - сбор и обработка материала, поиск и анализ литературы. Все авторы прочли и одобрили финальную версию статьи перед публикацией, согласны нести ответственность за все аспекты работы и гарантируют, что ими надлежащим образом были рассмотрены и решены вопросы, связанные с точностью и добросовестностью всех частей работы.

\section{Литература / References}

1. Тоноян АС, Агеев ИС, Овчаренко ТА, Захарова НЕ, Александрова EВ, Горяйнов СА, Быканов AЕ, Шурхай ВА, Шульц ЕИ, Пронин ИН. Новые возможности магнитно-резонансной трактографии в нейрорадиологии: модель HARDI-CSD. Вестник Российского фонда фундаментальных исследований. 2016;2(90): 20-32. doi: 10.22204/2410-4639-2016-090-0220-32. [Tonoyan AS, Ageev IS, Ovcharenko TA, Zakharova NE, Alexandrova EV, Goryaynov SA, Bykanov AE, Shurkhay VA, Shults El, Pronin IN. [New Capabilities of Magnetic Resonance Tractography In Neuroradiology: HARDI-CSD Model]. Russian Foundation for Basic Research Journal. 2016;2(90):20-32. Russian. doi: 10.22204/2410-4639-2016-090-02-20-32.]

2. Ramnani N, Miall RC. A system in the human brain for predicting the actions of others. Nat Neurosci. 2004;7(1):85-90. doi: 10.1038/ nn1168.

3. Hofer S, Frahm J. Topography of the human corpus callosum revisited - comprehensive fiber tractography using diffusion tensor magnetic resonance imaging. Neuroimage. 2006;32(3):989-994. doi: 10.1016/j.neuroimage.2006.05.044

4. Jones DK, Knösche TR, Turner R. White matter integrity, fiber count, and other fallacies: the do's and don'ts of diffusion MRI. Neuroimage. 2013;73:239-254. doi: 10.1016/j.neuroimage.2012.06.081.

5. Farquharson S, Tournier JD, Calamante F, Fabinyi G, Schneider-Kolsky M, Jackson GD, Connelly A. White matter fiber tractography: why we need to move beyond DTI. J Neurosurg. 2013;118(6):1367-1377. doi: 10.3171/2013.2.JNS121294.

6. Jeurissen B, Leemans A, Tournier JD, Jones DK, Sijbers J. Investigating the prevalence of complex fiber configurations in white matter tissue with diffusion magnetic resonance imaging. Hum Brain Mapp. 2013;34(11):2747-2766. doi: 10.1002/hbm.22099.
7. Kramm CM, Wagner S, Van Gool S, Schmid H, Sträter R, Gnekow A, Rutkowski S, Wolff JE. Improved survival after gross total resection of malignant gliomas in pediatric patients from the HIT-GBM studies. Anticancer Res. 2006;26(5B):3773-3779.

8. Tuch DS, Reese TG, Wiegell MR, Makris N, Belliveau JW, Wedeen VJ. High angular resolution diffusion imaging reveals intravoxel white matter fiber heterogeneity. Magn Reson Med. 2002;48(4):577-582. doi: 10.1002/mrm.10268.

9. Tournier JD, Calamante F, Connelly A. Robust determination of the fibre orientation distribution in diffusion MRI: non-negativity constrained super-resolved spherical deconvolution. Neuroimage. 2007;35(4):1459-1472. doi: 10.1016/j.neuroimage.2007.02.016.

10. Jeurissen B, Tournier JD, Dhollander T, Connelly A, Sijbers J. Multi-tissue constrained spherical deconvolution for improved analysis of multi-shell diffusion MRI data. Neuroimage. 2014;103:411-426. doi: 10.1016/j.neuroimage.2014.07.061.

11. Veraart J, Novikov DS, Christiaens D, AdesAron B, Sijbers J, Fieremans E. Denoising of diffusion MRI using random matrix theory. Neuroimage. 2016;142:394-406. doi: 10.1016/j. neuroimage.2016.08.016.

12. Tournier JD, Calamante F, Connelly A. Determination of the appropriate $b$ value and number of gradient directions for high-angular-resolution diffusion-weighted imaging. NMR Biomed. 2013;26(12):1775-1786. doi: 10.1002/ nbm.3017.

13. Dhollander T, Connelly A. A novel iterative approach to reap the benefits of multi-tissue CSD from just single-shell $(+b=0)$ diffusion MRI data. Proc. Intl. Soc. Mag. Reson. Med. 24. 2016; 3010.

14. Binder DK, Sonne DC, Fischbein NJ. Cranial nerves: anatomy, pathology, imaging. New York: Thieme; 2010. 248 p.
15. Rubino PA, Rhoton AL Jr, Tong $X$, de Oliveira $E$. Three-dimensional relationships of the optic radiation. Neurosurgery. 2005;57(4 Suppl):219-227; discussion 219-227. doi: 10.1227/01.neu.0000176415.83417.16.

16. Meyer A. The connections of the occipital lobes and the present status of the cerebral visual affections. Transactions Association of the American Physicians. 1907;(22):7-23.

17. Sincoff EH, Tan Y, Abdulrauf SI. White matter fiber dissection of the optic radiations of the temporal lobe and implications for surgical approaches to the temporal horn. J Neurosurg. 2004;101(5):739-746. doi: 10.3171/ jns.2004.101.5.0739.

18. Türe U, Yaşargil MG, Friedman AH, Al-Mefty O. Fiber dissection technique: lateral aspect of the brain. Neurosurgery. 2000;47(2):417-426; discussion 426-427. doi: 10.1097/00006123200008000-00028.

19. Ebeling U, Reulen HJ. Neurosurgical topography of the optic radiation in the temporal lobe. Acta Neurochir (Wien). 1988;92(1-4):29-36. doi: $10.1007 / \mathrm{BF} 01401969$.

20. Quinones-Hinojosa A, Raza SM, Ahmed I, Rincon-Torroella J, Chaichana K, Olivi A. Middle Temporal Gyrus Versus Inferior Temporal Gyrus Transcortical Approaches to High-Grade Astrocytomas in the Mediobasal Temporal Lobe: A Comparison of Outcomes, Functional Restoration, and Surgical Considerations. Acta Neurochir Suppl. 2017;124:159-164. doi: 10.1007/978-3-319-39546-3_25.

21. Dhollander T, Raffelt D, Connelly A. Unsupervised 3-tissue response function estimation from single-shell or multi-shell diffusion MR data without a co-registered T1 image [Internet]. ResearchGate. 2016. Available from: https://www.researchgate.net/publication/307863133_Unsupervised_3-tissue_response_function_estimation_from_single-shell_or_multi-shell_diffusion_MR_data_ without_a_co-registered_T1_image. 


\title{
Magnetic resonance tractography based on the constrained spherical deconvolution in patients with gliomas of the optic pathway
}

\author{
A.A. Baev • E.L. Pogosbekian' • N.E. Zakharova' • \\ D.I. Pitskhelauri' • A.I. Batalov' • A.M. Shkatova' • E.I. Shults' • \\ A.E. Bykanov' • S.A. Maryashev' • T.A. Konakova' • I.N. Pronin'
}

Background: The use of magnetic resonance (MR) tractography in neurosurgery is becoming an increasingly common practice for noninvasive imaging of white matter pathways. The most common method of tract reconstruction is the deterministic algorithm of diffusion tensor magnetic resonance imaging (MRI). However, this method of reconstructing pathways has a number of significant limitations. The most important of them are the lack of the possibility of visualizing the intersecting fibers, the complexity of building tracts in the area of perifocal edema and in the immediate vicinity of the tumor borders. The method of MR tractography, based on obtaining a diffusion image with a high angular resolution (High Angular Resolution Diffusion Imaging, HARDI), using the constrained spherical deconvolution (CSD) algorithm for post-processing of data, makes it possible to avoid these disadvantages. Relatively recently, a new algorithm, Single-Shell 3-Tissue CSD (SS3T(SD), has been proposed for processing HARDI data, which has the potential to improve the reconstructing of pathways in the area of perifocal edema or edema-infiltration.

Aim: To evaluate the potential of the new SS3TCSD algorithm compared to ST-CSD (Single-Tissue (SD) in the imaging of the optic radiation and visual tracts in patients with gliomas.

Materials and methods: Diffusion and routine brain MRI was performed in 10 patients with newly diagnosed cerebral gliomas, followed by reconstruction of the optic radiation and visual tracts. We compared new algorithms for postprocessing MR tractography (ST-CSD and SS3TCSD) in imaging of the optic tract and visual radiation in patients with brain gliomas affecting various parts of the visual system.

Results: The SS3T-CSD method showed a lower mean percentage of false positive tracts compared to the ST-CSD method: $19.75 \%$ for the SS3T-CSD method and $80.32 \%$ for the ST-CSD method in cases of proximity of the tumor to the tracts, $5.27 \%$ for the SS3T-CSD method and $25.27 \%$ for the STCSD method in cases of reconstructing tracts in healthy white matter.

Conclusion: The SS3T-CSD method has a number of advantages over ST-CSD and allows for successful imaging of the optic pathways that have a complex structure and repeatedly change direction along their course.

Key words: magnetic resonance tractography, optic radiation, optic tract, CSD-HARDI

For citation: Baev AA, Pogosbekian EL, Zakharova NE, Pitskhelauri DI, Batalov Al, Shkatova AM, Shults EI, Bykanov AE, Maryashev SA, Konakova TA, Pronin IN. Magnetic resonance tractography based on the constrained spherical deconvolution in patients with gliomas of the optic pathway. Almanac of Clinical Medicine. 2021;49(1):11-20. doi: 10.18786/20720505-2021-49-009.

Received 22 December 2020; revised 17 February 2021; accepted 19 February 2021; published online 12 March 2021

\section{Conflict of interests}

The authors declare that there is no conflict of interests regarding the publication of this article.

\section{Authors' contributions}

A.A. Baev, the study concept and design, data management, analysis of the results, literature search and analysis, text writing E.L. Pogosbekian, analysis of the results, statistical analysis, literature search and analysis, text writing; N.E. Zakharova, the paper concept and design, analysis and interpretation of the study results, text writing and editing, approval of the final version of the manuscript; D.I. Pitskhelauri, the paper concept and design, data collection and management, analysis of the results; A.I. Batalov, data collection and management, text editing; A.M. Shkatova, data collection and management, literature search and analysis, text writing; E.I. Shults, A.E. Bykanov, S.A. Maryashev and T.A. Konakova, data collection and management, literature search and analysis; I.N. Pronin, the study concept and design, text editing, approval of the final version of the manuscript. All the authors have read and approved the final version of the manuscript before submission, agreed to be accountable for all aspects of the work in ensuring that questions related to the accuracy or integrity of any part of the work are appropriately investigated and resolved.

Alexander A. Baev - Radiologist, Department of Neuroradiology and Radioisotope Diagnostic Imaging'; ORCID: https://orcid.org/0000-0003-49080534

$\triangle 16$ 4-ya Tverskaya-Yamskaya ul., Moscow, 125047, Russian Federation. E-mail: abaev@nsi.ru Eduard L. Pogosbekian - Medical Physicist, Department of Neuroradiology and Radioisotope Diagnostic Imaging '; ORCID: https://orcid.org/00000002-4803-6948. E-mail: epogosbekyan@nsi.ru

Natalia E. Zakharova - MD, PhD, Professor of Russ. Acad. Sci., Leading Research Fellow, Department of Neuroradiology and Radioisotope Diagnostic Imaging ${ }^{1}$; ORCID: https://orcid.org/0000-0002-05163613. E-mail: nzakharova@nsi.ru

David I. Pitskhelauri - MD, PhD, Leading Research Fellow, Head of Neurosurgical Department ${ }^{1}$; ORCID: https://orcid.org/0000-0003-0374-7970.

E-mail: dav@nsi.ru

Artem I. Batalov - MD, PhD, Junior Research Fellow, Department of Neuroradiology and Radioisotope Diagnostic Imaging '; ORCID: https://orcid.org/00000002-8924-7346. E-mail: abatalov@nsi.ru

Anastasia M. Shkatova - Neurosurgeon, Neurosurgical Department'; ORCID: https://orcid. org/0000-0003-4957-4286.

E-mail: shkatova.a.m.v@gmail.com

Evgeny I. Shults - MD, PhD, Radiologist, Department of Neuroradiology and Radioisotope Diagnostic Imaging '; ORCID: https://orcid.org/00000001-5406-944X.E-mail:evgshults@gmail.com

Andrey E. Bykanov - MD, PhD, Neurosurgeon, Neurosurgical Department ${ }^{1}$; ORCID: https://orcid. org/0000-0002-0588-4779.

E-mail: abykanov7@gmail.ru

Sergey A. Maryashev-MD, PhD, Neurosurgeon, Neurosurgical Department '; ORCID: https://orcid. org/0000-0002-0108-0677. E-mail: smaryashev@gmail.com

Tatiana A. Konakova - Postgraduate Student, Department of Neuroradiology and Radioisotope Diagnostic Imaging '; ORCID: https://orcid.org/00000002-2505-7981.E-mail: tknsipet@gmail.com

Igor N. Pronin - MD, PhD, Professor, Member of Russ. Acad. Sci., Head of Department of Neuroradiology and Radioisotope Diagnostic Imaging'; ORCID: https://orcid.org/0000-0002-44800275.E-mail:pronin@nsi.ru

N.N. Burdenko National Medical Research Center of Neurosurgery; 16 4-ya Tverskaya-Yamskaya ul., Moscow, 125047, Russian Federation 